\title{
Is the anisotropy of perceived 3-D shape invariant across scale?
}

\author{
JACK M. LOOMIS \\ University of California, Santa Barbara, California \\ and \\ JOHN W. PHILBECK \\ Camegie Mellon University, Pittsburgh, Pennsylvania
}

\begin{abstract}
A number of studies have resulted in the finding of a 3-D perceptual anisotropy, whereby spatial intervals oriented in depth are perceived to be smaller than physically equal intervals in the frontoparallel plane. In this experiment, we examined whether this anisotropy is scale invariant. The stimuli were L shapes created by two rods placed flat on a level grassy field, with one rod defining a frontoparallel interval, and the other, a depth interval. Observers monocularly and binocularly viewed $L$ shapes at two scales such that they were projectively equivalent under monocular viewing. Observers judged the aspect ratio (depth/width) of each shape. Judged aspect ratio indicated a perceptual anisotropy that was invariant with scale for monocular viewing, but not for binocular viewing. When perspective is kept constant, monocular viewing results in perceptual anisotropy that is invariant across these two scales and presumably across still larger scales. This scale invariance indicates that the perception of shape under these conditions is determined independently of the perception of size.
\end{abstract}

In recent years, a number of experimental studies have shown that, even under full-cue viewing, there is a clear anisotropy of perceived 3-D shape, with spatial intervals having a significant depth component appearing shorter than equal lengths oriented within a frontoparallel plane (Amorim, Loomis, \& Fukusima, 1998; Baird \& Biersdorf, 1967; Levin \& Haber, 1993; Loomis, Da Silva, Fujita, \& Fukusima, 1992; Norman, Todd, Perotti, \& Tittle, 1996; Ribeiro-Filho, Fukusima, \& Da Silva, 1995; Tittle, Todd, Perotti, \& Norman, 1995; Todd, Tittle, \& Norman, 1995; Toye, 1986; Wagner, 1985; Yang, Wade, \& Proffitt, 1997); these recent studies confirm earlier studies showing systematic misperception of 2-D shapes viewed with large values of slant under full cues (e.g., Beck \& Gibson, 1955; Joynson \& Newson, 1962; Kaiser, 1967; Wallach \& Moore, 1962; see Sedgwick, 1986, for a review of much of this earlier work).

It might be thought that this anisotropy of perceived 3-D shape is the consequence of some compressive nonlinearity between physical and perceived egocentric distance; such a nonlinear psychophysical mapping would imply, for instance, that depth intervals on the ground surface $^{1}$ would be seen as increasingly foreshortened rel-

This research was supported by ONR Grant N00014-95-1-0573. The data were reported at the 1996 annual meeting of the Association for Research in Vision and Ophthalmology. The authors thank Jim Todd, Hal Sedgwick, and an anonymous reviewer for helpful comments on an earlier version of the manuscript, and Shira Brill for her assistance with the experiment. Correspondence should be addressed to J. M. Loomis, Department of Psychology, University of California, Santa Barbara, CA 93106-9660 (e-mail: loomis@psych.ucsb.edu). ative to frontoparallel intervals, the more distant the intervals are from the observer. Evidence for such a compressive nonlinearity has been reported in a number of studies (e.g., see review by Sedgwick, 1986); in particular, Gilinsky (1951) found that perceived distance was a hyperbolic function of physical distance with an asymptotic perceived distance of $29 \mathrm{~m}$. However, the depth scaling literature as a whole is equivocal about whether the psychophysical mapping is compressively nonlinear, with other studies suggesting that the mapping is linear and still others suggesting that perceived distance might even be an accelerating function of physical distance (see reviews by Baird, 1970; Da Silva, 1985; Sedgwick, 1986). Moreover, other evidence based on a variety of motoric responses indicates that perceived egocentric distance under full-cue viewing conditions is both linear and accurate, at least up to 15-20 m (Elliott, 1986, 1987; Fukusima, Loomis, \& Da Silva, 1997; Loomis et al., 1992; Loomis, Klatzky, Philbeck, \& Golledge, 1998; Philbeck \& Loomis, 1997; Philbeck, Loomis, \& Beall, 1997; Rieser, Ashmead, Talor, \& Youngquist, 1990; Steenhuis \& Goodale, 1988; Thomson, 1983). Loomis and his colleagues (Loomis et al., 1992; Loomis, Da Silva, Philbeck, \& Fukusima, 1996) interpret these two results, the anisotropy of perceived 3-D shape and the accuracy of perceived egocentric distance up to $15 \mathrm{~m}$, in terms of a dissociation between the perception of spatial extent and the perception of the absolute locations specifying the extent.

In one of the studies above (Loomis et al., 1992, Experiments 1 and 2), observers were asked to adjust a depth interval on the ground, by way of instructions to an assistant, until it was judged physically equal to a width in- 
terval on the ground ("objective" instructions). Both the depth and width intervals were defined by highly visible targets placed on the ground. Observers systematically made the depth interval (in the median plane) physically longer than the width interval (in a frontoparallel plane) in attempting to match the two in length. This result, along with those of the above-cited studies, is strong evidence of 3-D perceptual anisotropy, even under full-cue viewing. Moreover, this anisotropy was found by Loomis et al. (1992) to increase with egocentric distance of the stimulus configuration, as was indicated by the observers' making the physical depth interval even larger at far distance in order to produce an apparent match with the width interval of constant size.

A limitation of the matching tasks used by Loomis et al. (1992) is that they confounded egocentric distance of the configuration with its local surface orientation or optical slant $^{2}$ (i.e., more distant shapes on the surface plane have larger slant values). In an important paper, Levin and Haber (1993) have shown that the judged length of large spatial intervals on the ground is influenced by their angular extents, all else being equal. The question arises whether the dependence of perceptual anisotropy on egocentric distance (Loomis et al., 1992) is a consequence of the changing projective sizes of the two intervals or of some other concomitant of egocentric distance.

Of relevance to the issue being addressed here is the experience of the first author when flying over the highly textured plains of the midwestern United States under conditions of very high visibility. If the hypothesis proposed by Gilinsky (1951) of a hyperbolic psychophysical function with an asymptotic $29 \mathrm{~m}$ should be correct, the textured ground plane ought to have appeared equidistant (i.e., a spherical surface concave with respect to the observer). Yet, the impression was that of a clearly receding planar surface, very much like that which one has when looking obliquely at the textured surface of a table from a standing position. In a similar vein, Gibson (1950, p. 4) noted that a textured field viewed from an elevation "appears to extend to a great distance."

When one considers two spatial layouts differing only in scale and viewed from appropriate locations so that they are projectively equivalent (for monocular vision), it is apparent that many of the monocular cues of relative distance (e.g., relative size, texture gradient, linear perspective, shape from shading) specify only the ratios of exocentric distances (Cutting \& Vishton, 1995); that is, they specify object shape independently of scale. This raises the possibility that beyond some near distance, two spatial layouts differing in scale will be perceived equivalently in terms of shape. In particular, the perceptual anisotropy associated with objects on the surface of a planar surface will be scale invariant.

The experiment reported here simultaneously addresses the two related questions raised above: whether perceived spatial layout is scale invariant, and whether optical slant, rather than some other concomitant of egocentric distance, accounts for the perceptual anisotropy. In this ex- periment, observers viewed $\mathrm{L}$ shapes, defined by pairs of rods at right angles, and made judgments of their aspect ratios. The experiment was performed at two scales, differing by a factor of 3 , such that corresponding L shapes at the two scales were projectively equivalent (for monocular viewing). ${ }^{3}$ We actually conducted the experiment under both monocular and binocular viewing, but were more interested in the comparison under monocular viewing, for it was in this condition that the relative distance cues were very nearly the same at the two scales. The presence of above-threshold binocular disparity information in the binocular viewing conditions means that the relative distance cues differed slightly at the two scales.

\section{METHOD}

\section{Observers}

Sixteen adult observers ( 8 men and 8 women) were recruited from the university community and paid for their participation. Four men and 4 women were randomly assigned to participate in the monocular viewing group, with the rest participating in the binocular viewing group. Their ages ranged from 19 to 40 years; the median age was 21 years. One observer in the monocular condition was amblyopic in the covered eye. All observers were naive about the purpose of the experiment.

\section{Stimuli and Design}

The experiment took place outdoors in a level, grassy field (approximately $30 \times 100 \mathrm{~m}$ ), which adjoined a campus building with an outdoor staircase. Sidewalks, trees, buildings, and passers-by were visible from the observation locations. In addition to the between-group monocular/binocular manipulation, we also varied the scale of the stimulus configurations (within group): the "largescale" configurations were three times larger and three times more distant than the "small-scale" configurations; the observer's eye height was also three times higher at the large scale. In this way, the optical slants (or angles of regard) were matched at the two scales, yielding projectively equivalent stimuli. The eye height manipulation was blocked and counterbalanced so that half the observers in each group saw the small scale stimuli first, and the other half, the large scale first. Eye height was manipulated by having the observer stand at specified locations on the staircase: either near the bottom (eye height, $195 \mathrm{~cm}$ ) or at the top (eye height, $585 \mathrm{~cm}$ ). The observers stood on boards of appropriate thickness to compensate for individual differences in normal eye height. On each trial within a scale block, a stimulus configuration appeared at one of three possible distances (small scale, 3.9, 5.2, and $6.5 \mathrm{~m}$; large scale, 11.7, 15.6 , and $19.5 \mathrm{~m}$ ). This was the distance measured along the ground from a point directly below the observer's eyes to the nearest point in the stimulus configuration. The distance manipulation was blocked and counterbalanced. The stimuli themselves were cylindrical rods arranged in an $L$ shape and placed flat on the field. One rod defined a frontal interval, and the other, a depth interval. There were two sets of rods, differing in scale: The small-scale rods had a diameter of about $1 \mathrm{~cm}$, and the large-scale rods were three times larger. The small-scale rods had thin spikes inserted perpendicularly at either end. These spikes were inserted into the ground to hold the rods in place and were generally not visible. The length of the frontal rod did not vary at each scale (small scale, $30.5 \mathrm{~cm}$; large scale, $91.4 \mathrm{~cm}$ ). The lengths of the depth rods were chosen in such a way that they created aspect ratios ranging from 0.5 to 2.5 (depth/width) with respect to the frontal rods, in increments of 0.25 .

For our analysis, we were really only interested in two of the stimulus aspect ratios (1.5:1 and 2:1, depth/width); for each subject, these were presented twice at each viewing distance. The other 
stimulus aspect ratios were employed in the experiment in order to reduce possible expectancy effects among the observers. Thus, these were randomly interspersed among the trials above, there being an equal number of trials with stimuli drawn from these other values. These trials were not analyzed but were included to minimize the possibility that observers would assume that they were seeing the same range of stimuli in the two scales. For each observer, scale, and distance, four aspect ratios were randomly chosen from the set of seven nonexperimental aspect ratios. Thus, the means of the set of experimental and non-experimental aspect ratios were generally different across the large and small scales, both between scales for each observer and also between observers.

\section{Procedure}

The observers saw a single $\mathrm{L}$ shape at a time and gave a verbal judgment of its aspect ratio (depth/width) under objective instructions (Carlson, 1977); see Figure 1. The instructions indicated that the observer should imagine standing directly over the shape and compare the two rods as if using a tape measure. Between trials, the observer turned away from the field while the experimenter positioned the next stimulus. A small carpenter's level was used to verify that each rod was indeed level.

\section{RESULTS}

The data from 3 observers were excluded from the analysis because these observers appeared to be using the response measure inconsistently or because they repeatedly expressed confusion about the task. For our response measure, we use the ratio of aspect ratios (estimated aspect ratio/physical aspect ratio). This measure permits a direct comparison of the responses to stimuli with different physical ratios and at different scales (see Figure 1). In Figure 2, the average ratio of ratios is plotted as a function of distance to the target configuration (expressed in units of eye height to provide a common abscissa between the small and large scales). Each panel also gives the predicted response measures if there were no perceptual anisotropy (ratio of ratios equal to 1.0) and if the perceptual shape were equal to retinal shape (indicated by the small triangles).

As can be seen in Figure 2, there was very substantial perceptual anisotropy for both monocular and binocular

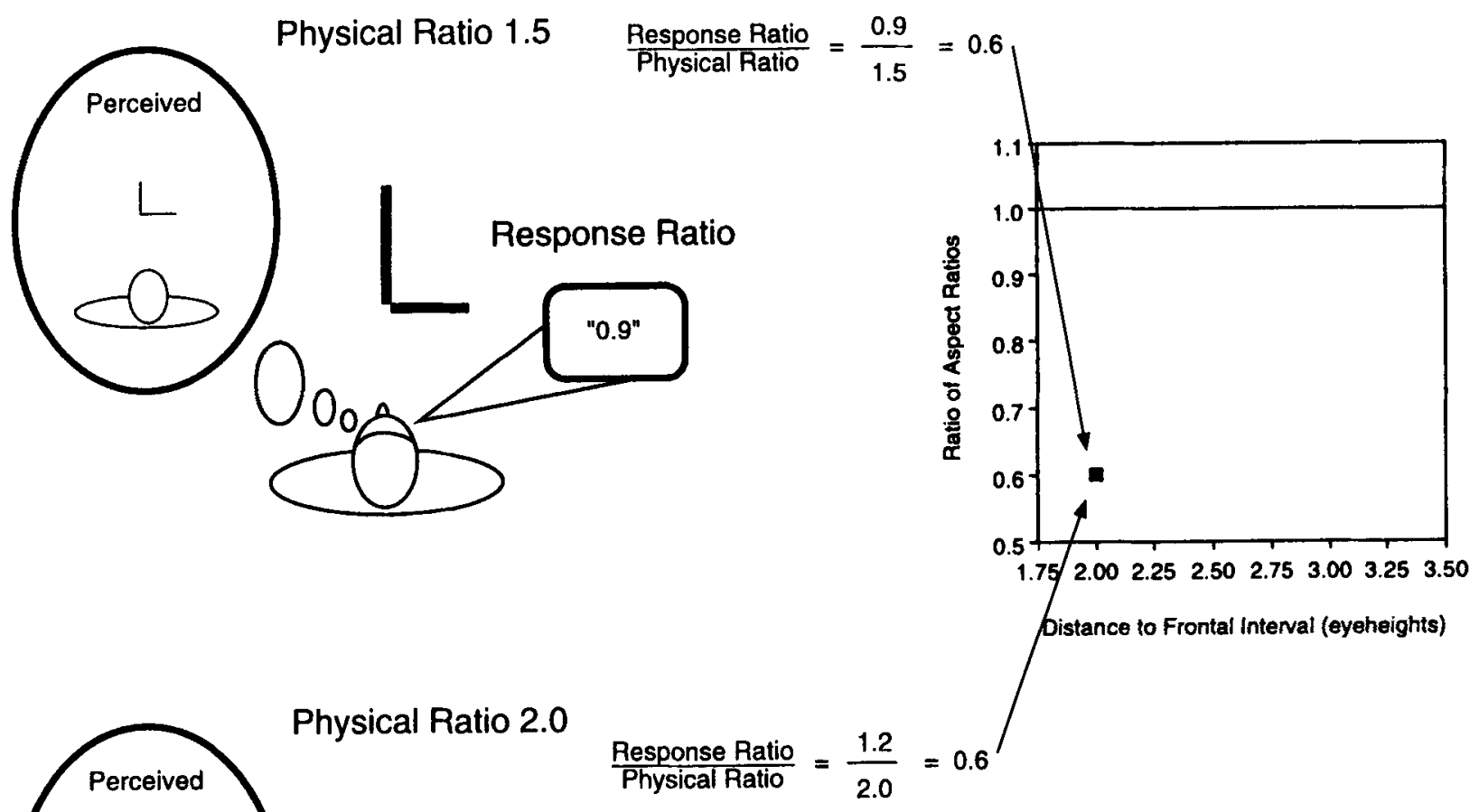

Response Ratio

Figure 1. Representation of how the response ratio was obtained. For L-shaped figures having an aspect ratio (depth divided by width) of either 1.5 or 2.0 , the observer judged the perceived aspect ratio. The response measure was the ratio of judged aspect ratio to physical aspect ratio. Veridical perception together with accurate judgment ought to result in a response ratio of 1.0 . 

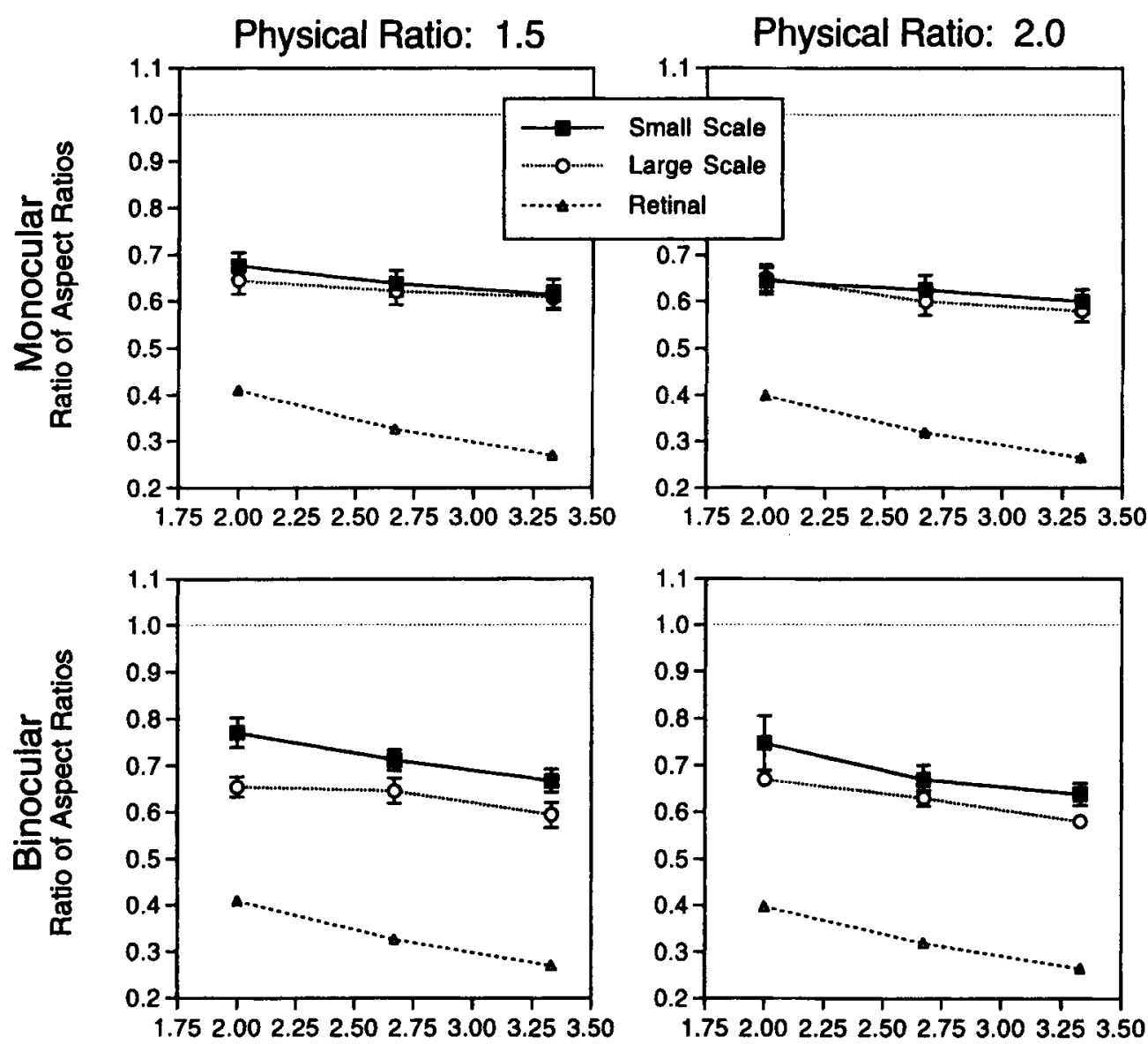

\section{Distance to Frontal Interval (eyeheights)}

Figure 2. Results of the experiment.

viewing, although not as extreme as that predicted by the aspect ratios of the retinal images. The dependent measure (ratio of aspect ratios) was subjected to separate analyses of variance for monocular and binocular viewing. Increasing the scale by a factor of three had virtually no effect on perceptual anisotropy under monocular viewing $[F(1,7)=0.69, p=.43]$; the only reliable effect was that of distance $[F(2,14)=15.87, p<.001]$. In contrast, there was an effect of scale for binocular viewing $[F(1,7)=8.50, p<.05]$, with less anisotropy evident at the smaller scale; in addition, there was a main effect of distance $[F(2,14)=7.61, p<.01]$.

\section{DISCUSSION}

For the two scales at which we conducted the experiment, the dependence of perceptual anisotropy on distance to the configuration, expressed in eye heights, was invariant for monocular viewing (Figure 2). In contrast, with binocular viewing, the perceptual anisotropy was reduced, but only slightly, for the smaller scale (Figure 2), probably because binocular disparity was slightly more effective. In earlier work (Loomis \& Philbeck, 1994), we found that even for monocular viewing, the anisotropy was greater for target distances of $400 \mathrm{~cm}$ than for target distances of $60 \mathrm{~cm}$, presumably because variations in monocular parallax (the stimulus to accommodation) were more effective at the smaller scale.

Because monocular parallax ceases to be effective for distances beyond several meters, the invariant perceptual anisotropy obtained here for monocular viewing is probably that which would be observed at still larger scales (e.g., viewing from the top of tall building, from the top of a mountain, or from a spacecraft in low earth orbit), provided that the relative distance information conveyed by the various relative cues (relative size, texture gradient, linear perspective, etc.) is the same across scales. Even with binocular viewing, it is likely that perceptual anisotropy is invariant with scale beyond target distances of $20 \mathrm{~m}$ because binocular disparity quickly falls off in effectiveness with distance (at least for targets that are small relative to their egocentric distances, as they are here).

The results suggest that the variation in perceived target shape with target distance (Figure 2) is mostly determined by optical slant (as specified by the various perspective cues), at least for the distances studied here. In 
contrast, for targets within $2 \mathrm{~m}$, binocular disparity is also a major determinant of perceived shape, such that there is much less perceptual anisotropy, a result observed by Loomis and Philbeck (1994). For larger distances beyond the effective range of binocular disparity, the perceived aspect ratio ought to be solely a function of optical slant, as specified by the various perspective cues.

In the present study, we did not have observers judge the sizes of the targets at the two scales, but the reader can rest assured that targets at the two scales looked very different in size. Assuming this, an important conclusion is that the perception of shape under these conditions is determined independently of the perception of size. Thus, two 3-D configurations varying in scale (e.g., a large building viewed from the air and a scale model of the same viewed from a standing position) can appear identical in terms of shape, as specified by perspective cues, but vastly different in size, by virtue of independent cues to size and egocentric distance.

Now consider the following thought experiment, currently in the planning stages. Observers judge both the perceived distances and perceived shapes of identical Lshaped targets placed on the horizontal tops of visible pedestals that vary in height from near zero to just under the observer's eye height. The observers view these targets, all $3 \mathrm{~m}$ away, under full cues. The present results along with the slant/shape literature indicate that the perceptual anisotropy of the targets will become more extreme as optical slant increases with elevation. The distance perception literature indicates that the perceived distances of the targets under these full-cue conditions will be very nearly equal to each other and also quite accurate. This important conclusion can be drawn: The variation in perceived shape with target elevation while physical and perceived distance are held constant means that the perception of spatial extent is somewhat independent of the perception of location; specifically, the perception of extent depends on more than the perceived locations of the endpoints specifying the extent (Loomis et al., 1992; Loomis et al., 1996; Loomis \& Philbeck, 1994; for a similar argument involving the two dimensions of direction, see Abrams \& Landgraf, 1990; Gillam \& Chambers, 1985; Mack, Heuer, Villardi, \& Chambers, 1985). This dissociation between perceived extent and perceived location means that the enterprise of using perceived exocentric distances to construct a scale of perceived egocentric distance (e.g., Gilinsky, 1951) is questionable.

Finally, a few words about perceived egocentric distance are in order. Although a number of the studies alluded to earlier have indicated that perceived egocentric distance is linear in physical distance out to $20 \mathrm{~m}$ and beyond, it certainly must be the case that, for a person standing on a flat plain, the perceived distance of targets near the ground surface must eventually asymptote, as Gilinsky (1951) maintained, because there is insufficient visual information to specify distances on the order of kilometers. However, the first author's observation of how the ground surface appears from a high flying aircraft suggests that perceived egocentric distances of tar- gets on the ground vary considerably more when viewed from high up than when viewed from near the ground. Apparently, the ratios of egocentric distances to locations on the surface are determined by the perspective cues, while the perceived scale of the surface is established by other cues, such as familiar size. The perceived egocentric distance of a particular target is then determined by its perceived relation to the surface. If true, this speculative account means that there must be a complex interplay between the processes underlying the perception of relative distance, the perception of scale, the perception of location, and the perception of local shape.

\section{REFERENCES}

Abrams, R. A., \& LANDGRaF, J. Z. (1990). Differential use of distance and location information for spatial localization. Perception \& Psychophysics, 47, 349-359.

Amorim, M.-A.. LoOmis, J. M., \& Fukusima, S. S. (1998). Reproduction of object shape is more accurate without the continued availability of visual information. Perception, 27, 69-86.

BaIRD, J. C. (1970). Psychophysical analysis of visual space. Oxford: Pergamon Press.

BAIRD, J. C., \& BIERSDORF, W. R. (1967). Quantitative functions for size and distance judgments. Perception \& Psychophysics, 2, 161-166.

BECK, J., \& GiBson, J. J. (1955). The relation of apparent shape to apparent slant in the perception of objects. Journal of Experimental Psychology, 50, 125-133.

CarLson, V. R. (1977). Instructions and perceptual constancy judgments. In W. Epstein (Ed.), Stability and constancy in visual perception: Mechanisms and processes (pp. 217-254). New York: Wiley.

Cutting, J. E., \& Vishton, P. M. (1995). Perceiving layout and knowing distances: The integration, relative potency, and contextual use of different information about depth. In W. Epstein \& S. Rogers (Eds.), Perception of space and motion (pp. 69-117). New York: Academic Press.

DA Silva, J. A. (1985). Scales for perceived egocentric distance in a large open field: Comparison of three psychophysical methods. American Journal of Psychology, 98, 119-144.

ElliotT, D. (1986). Continuous visual information may be important after all: A failure to replicate Thomson (1983). Journal of Experimental Psychology: Human Perception \& Performance, 12, 388-391.

ELLIOTT, D. (1987). The influence of walking speed and prior practice on locomotor distance estimation. Journal of Motor Behavior, 19, 476-485.

Fukusima, S. S., Loomis, J. M., \& Da Silva, J. A. (1997). Visual perception of egocentric distance as assessed by triangulation. Journal of Experimental Psychology: Human Perception \& Performance, 23, 86-100.

Gibson, J. J. (1950). The perception of the visual world. Boston: Houghton-Mifflin.

GibSon, J. J., \& CoRnsweEt, J. (1952). The perceived slant of visual surfaces-optical and geographical. Journal of Experimental Psychology, 44, 11-15.

GILINSKY, A. S. (1951). Perceived size and distance in visual space. Psychological Review, 58, 460-482.

Gillam, B., \& Chambers, D. (1985). Size and position are incongruous: Measurements on the Müller-Lyer figure. Perception \& Psychophysics, 37, 549-556.

JoYNSON, R. B., \& NEwSON, L. J. (1962). The perception of shape as a function of inclination. British Journal of Psychology, 53, 1-15.

KAISER, P. K. (1967). Perceived shape and its dependency on perceived slant. Journal of Experimental Psychology, 75, 345-353.

LEVIN, C. A., \& HABER, R. N. (1993). Visual angle as a determinant of perceived interobject distance. Perception \& Psychophysics, 54, 250-259.

Loomis, J. M., Da Silva, J. A., FujtTa, N., \& Fukusima, S. S. (1992). Visual space perception and visually directed action. Journal of Experimental Psychology: Human Perception \& Performance, 18, 906-921. 
LoOmis, J. M., Da Silva, J. A., Philbeck, J. W., \& Fukusima, S. S. (1996). Visual perception of location and distance. Current Directions in Psychological Science, 5, 72-77.

Loomis, J. M., Klatzky, R. L., Philbeck, J. W., \& Golledge, R. G. (1998). Assessing auditory distance perception using perceptually directed action. Perception \& Psychophysics, 60, 966-980.

LOOMIS, J. M., \& PHILBECK, J. W. (1994, November). Distortion of visual space under full cues is not scale-invariant. Paper presented at the meeting of the Psychonomic Society, St. Louis.

MaCK, A., Heuer, F., Villardi, K., \& Chambers, D. (1985). The dissociation of position and extent in Müller-Lyer figures. Perception \& Psychophysics, 37, 335-344.

Norman, J. F., Todd, J. T., Perotti, V. J., \& Tittle, J. S. (1996). The visual perception of three-dimensional length. Journal of Experimental Psychology: Human Perception \& Performance, 22, 173-186.

PHILBECK, J. W., \& LoOMIs, J. M. (1997). Comparison of two indicators of perceived egocentric distance under full-cue and reduced-cue conditions. Journal of Experimental Psychology: Human Perception \& Performance, 23, 72-85.

Philbeck, J. W., Loomis, J. M., \& Beall, A. C. (1997). Visually perceived location is an invariant in the control of action. Perception \& Psychophysics, 59, 601-612.

Ribeiro-Filho, N. P., Fukusima, S. S., \& Da Silva, J. A. (1995, November). Size and distance perception in an environmental layout. Paper presented at the meeting of the Psychonomic Society, Los Angeles.

Rieser, J. J., Ashmead, D. H., Talor, C. R., \& Youngquist, G. A. (1990). Visual perception and the guidance of locomotion without vision to previously seen targets. Perception, 19, 675-689.

SEDGWICK, H. A. (1986). Space perception. In K. R. Boff, L. Kaufman, \& J. P. Thomas (Eds.), Handbook of perception and human performance: Vol. 1. Sensory processes and perception (pp. 21.1-21.57). New York: Wiley.

Steenhuis, R. E., \& Goodale, M. A. (1988). The effects of time and distance on accuracy of target-directed locomotion: Does an accurate short-term memory for spatial location exist? Journal of Motor Behavior, 20, 399-415.

Thomson, J. A. (1983). Is continuous visual monitoring necessary in visually guided locomotion? Journal of Experimental Psychology: Human Perception \& Performance, 9, 427-443.

Tittle, J. S., TodD, J. T., Perotti, V. J., \& Norman, J. F. (1995). Systematic distortion of perceived three-dimensional structure from motion and binocular stereopsis. Journal of Experimental Psychology: Human Perception \& Performance, 21, 663-678.
TodD, J. T., Tittle, J. S., \& Norman, J. F. (1995). Distortions of threedimensional space in the perceptual analysis of motion and stereo. Perception, 24, 75-86.

ToYe, R. C. (1986). The effect of viewing position on the perceived layout of space. Perception \& Psychophysics, 40, 85-92.

Wagner, M. (1985). The metric of visual space. Perception \& Psychophysics, 38, 483-495.

Wallach, H., \& MoORE, M. E. (1962). The role of slant in the perception of shape. American Journal of Psychology, 75, 285-293.

Yang, T. L., Wade, M. N., \& Proffitt, T. L. (1997, November). Heights are overestimated more in real and immersive environments than in pictures. Paper presented at the meeting of the Psychonomic Society, Philadelphia.

\section{NOTES}

1. Strictly speaking, a depth interval is one that is aligned with the eye or with the binocular egocenter. However, we refer to intervals that are both on the ground plane and in the median plane of the observer as depth intervals, even though they have both depth and frontoparallel components.

2. Optical slant is the angle between the normal to a local surface patch and the line of sight from the eye to its center (Gibson \& Cornsweet, 1952). The optical slant of the horizontal ground plane varies continuously from the point directly beneath the observer (where optical slant is zero) to the horizon (where it is $90^{\circ}$ ). Optical slant is independent of gaze direction, which corresponds to the primary line of sight.

3. In the case of monocular viewing, the experiment might appear to be unnecessary, for it might be thought that when monocular perspective is matched at the two scales, the perceived configurations must be the same. In an earlier study (Loomis \& Philbeck, 1994) involving matched perspective at two very small scales (table top and floor), the perceived shapes proved to be different, even for monocular viewing, suggesting perhaps that some relative distance cue, such as spatial variation in monocular parallax (the stimulus to accommodation), might have been more effective for the table top condition. Even in the present experiment where the two scales are sufficiently large to rule out any possible contribution of monocular parallax, one can still imagine that perceived scale, acting through the observer's prior experience, could influence perceived shape even with perspective cues held constant.

(Manuscript received August 25, 1997; revision accepted for publication March 10, 1998.) 